\title{
AN INFINITELY DIVIIBLE DISTRIBUTION INVOLVING MODIFIED BESSEL FUNCTIONS
}

\author{
MOURAD E. H. ISMAII ${ }^{1}$ AND KENNETH S. MILLER
}

ABSTRACT. We prove that the function

$$
\left(\frac{b}{a}\right)^{\mu-\nu} \frac{K_{\mu}\left(b x^{1 / 2}\right) K_{\nu}\left(a x^{1 / 2}\right)}{K_{\mu}\left(a x^{1 / 2}\right) K_{\nu}\left(b x^{1 / 2}\right)}
$$

is the Laplace transform of an infinitely divisible probability distribution when $\nu>\mu \geq 0$ and $b>a>0$. This implies the complete monotonic ity of the function. We also establish a representation as a Stieltjes transform, which implies in particular that the function has positive real part when $x$ lies in the right half-plane. We conjecture that

$$
\left(\frac{b}{a}\right)^{\mu-\nu} \frac{I_{\mu}\left(a x^{1 / 2}\right) I_{\nu}\left(b x^{1 / 2}\right)}{I_{\mu}\left(b x^{1 / 2}\right) I_{\nu}\left(a x^{1 / 2}\right)}
$$

also is the Laplace transform of an infinitely divisible probability distribution. It is known that in the limit as $\nu \rightarrow \infty$, the infinite divisibility property holds for both functions.

1. Introduction. A probability distribution on $[0, \infty)$ is said to be infinitely divisible if and only if for every natural number $n$, the $n$th root of its Laplace transform is a Laplace transform of a probability distribution. A function $f$ defined on $(0, \infty)$ and of class $C^{\infty}$ is said to be completely monotonic if $(-1)^{n} f^{(n)}(x) \geq 0$ on $(0, \infty)$ for all $n$. The connection between infinitely divisible distributions and completely monotonic functions is expressed in the following theorem (see $[5, \mathrm{p}$. 450]):

THEOREM 1. The function $w$ is the Laplace transform of an infinitely divisible probability distribution on $[0, \infty)$ if and only if $w=e^{-h}$ where $h(0+)=0$ and $h^{\prime}$ is completely monotonic.

In $§ 2$ we shall prove the following result:

THEOREM 2. Let $K_{\lambda}$ be the modified Bessel function of the second kind. Then

$$
F(x ; \mu, \nu)=\left(\frac{b}{a}\right)^{\mu-\nu} \frac{K_{\mu}\left(b x^{1 / 2}\right) K_{\nu}\left(a x^{1 / 2}\right)}{K_{\mu}\left(a x^{1 / 2}\right) K_{\nu}\left(b x^{1 / 2}\right)}
$$

Received by the editors July 6, 1981. Presented to the Society at the 1982 winter meeting on January 16, 1982.

1980 Mathematics Subject Classification. Primary 33A60, 60E07, 33A65; Secondary 60E10, 44A10, 44A20.

Key words and phrases. Modified Bessel functions, Laplace transform, Stieltjes transform, infinite divisibility.

${ }^{1}$ Research partially supported by NSF contract MCS81-02539

(c) 1982 American Mathematical Society 0002-9939/81/0000-1071/802.50 
is the Laplace transform of an infinitely divisible probability distribution when $\nu>$ $\mu \geq 0$ and $b>a>0$.

As $\nu$ increases without limit,

$$
K_{\nu}(x) \sim(2 \nu)^{\nu-1 / 2} e^{-\nu} x^{-\nu} \pi^{1 / 2}
$$

(see, for example [8]). Hence

$$
F(x ; \mu, \infty)=\left(\frac{b}{a}\right)^{\mu} \frac{K_{\mu}\left(b x^{1 / 2}\right)}{K_{\mu}\left(a x^{1 / 2}\right)} .
$$

The limiting case $(\nu \rightarrow \infty)$ of Theorem 2 was proved by Ismail and Kelker [9] using complex variable and special functions methods. Kent [11] and Wendel [14] independently encountered the same distribution $(\nu=\infty)$ in their studies of Bessel processes and Brownian motion, and established its infinite divisibility property by probabilistic methods. Ismail and May [10] provided an integral representation of the probability density function as the Laplace transform of a transcendental function. Miller [12, p. 82] proved that $F(x ; \mu, \nu)$ is monotone decreasing, a result needed in certain hypothesis testing problems.

In $\S 3$ we shall establish an integral representation of $F(x ; \mu, \nu)$ as a Stieltjes transform. This implies, for example, that $F(z ; \mu, \nu)$ has positive real part when $z$ lies in the right half-plane. The Stieltjes transform seems to play an important role in infinite divisibility problems, and in the theory of orthogonal polynomials (see $[2,3,9,10])$.

We conjecture that if $\nu>\mu \geq 0$ and $b>a>0$, then the function

$$
G(x ; \mu, \nu)=\left(\frac{b}{a}\right)^{\mu-\nu} \frac{I_{\mu}\left(a x^{1 / 2}\right) I_{\nu}\left(b x^{1 / 2}\right)}{I_{\mu}\left(b x^{1 / 2}\right) I_{\nu}\left(a x^{1 / 2}\right)}
$$

is also the Laplace transform of an infinitely divisible probability distribution; here $I_{\lambda}$ is the modified Bessel function of the first kind.

As $\lambda$ increases without limit

$$
I_{\lambda}(x) \sim\left(\frac{1}{2} x\right)^{\lambda} \Gamma^{-1}(\lambda+1)
$$

(see, for example [4, pp. 22 and 23]). Hence

$$
G(x ; \mu, \infty)=\left(\frac{b}{a}\right)^{\mu} \frac{I_{\mu}\left(a x^{1 / 2}\right)}{I_{\mu}\left(b x^{1 / 2}\right)}
$$

and the conjecture reduces to a known result, see $[9,10,14]$. In $\S 4$ we shall discuss this conjecture and formulate an equivalent conjecture involving functions that resemble theta functions.

2. Proof of Theorem 2. Our argument will be to show that

$$
h(x)=-\ln F(x ; \mu, \nu)
$$

has a completely monotonic derivative and that $h(0+)=0$. Then we may invoke Theorem 1 to complete the proof.

Clearly $h$ is well defined since $F(x ; \mu, \nu)$ is positive on $[0, \infty)$. Now (see $[1, p$. 375]) as $x \rightarrow 0+$,

$$
\begin{aligned}
& K_{\nu}(x) \sim \frac{1}{2} \Gamma(\nu)\left(\frac{1}{2} x\right)^{-\nu}, \quad \operatorname{Re} \nu>0 \\
& K_{0}(x) \sim-\ln x .
\end{aligned}
$$


This implies that $h(0+)=0$ since $F(0+; \mu, \nu)=1$. Using the recursion relation $[13$, p.79]

$$
K_{\nu}^{\prime}(x)=-K_{\nu-1}(x)-\nu K_{\nu}(x) / x
$$

we obtain

$$
\begin{aligned}
-2 x^{1 / 2} h^{\prime}(x)= & a \frac{K_{\mu-1}\left(a x^{1 / 2}\right)}{K_{\mu}\left(a x^{1 / 2}\right)}-b \frac{K_{\mu-1}\left(b x^{1 / 2}\right)}{K_{\mu}\left(b x^{1 / 2}\right)} \\
& -a \frac{K_{\nu-1}\left(a x^{1 / 2}\right)}{K_{\nu}\left(a x^{1 / 2}\right)}+b \frac{K_{\nu-1}\left(b x^{1 / 2}\right)}{K_{\nu}\left(b x^{1 / 2}\right)} .
\end{aligned}
$$

The integral representation [7]

$$
\frac{K_{\nu-1}\left(x^{1 / 2}\right)}{K_{\nu}\left(x^{1 / 2}\right)}=\frac{2}{\pi^{2}} \int_{0}^{\infty} \frac{x^{1 / 2} t^{-1}}{x+t}\left[J_{\nu}^{2}\left(t^{1 / 2}\right)+Y_{\nu}^{2}\left(t^{1 / 2}\right)\right]^{-1} d t, \quad \nu \geq 0
$$

where $J_{\nu}$ and $Y_{\nu}$ are the Bessel functions of the first and second kind, respectively, enables us to express $h^{\prime}$ in the form

$$
h^{\prime}(x)=\frac{b^{2}-a^{2}}{\pi^{2}} \int_{0}^{\infty}\left(x a^{2}+t\right)^{-1}\left(x b^{2}+t\right)^{-1} \phi(t) d t
$$

where

$$
\phi\left(t^{2}\right)=\left[J_{\mu}^{2}(t)+Y_{\mu}^{2}(t)\right]^{-1}-\left[J_{\nu}^{2}(t)+Y_{\nu}^{2}(t)\right]^{-1} .
$$

Now, Nicholson's formula [13, p. 444]

$$
J_{\lambda}^{2}(x)+Y_{\lambda}^{2}(x)=\frac{8}{\pi^{2}} \int_{0}^{\infty} K_{0}(2 x \sinh t) \cosh 2 \lambda t d t
$$

shows that $J_{\lambda}^{2}(x)+Y_{\lambda}^{2}(x)$ is a strictly increasing function of $\lambda$ for $\lambda \geq 0$ and $x$ a fixed positive number. Therefore $\phi(t)$ is strictly positive for $t>0$; and the integrand in equation (2.5) is a positive multiple of the completely monotonic function $\left(t+a^{2} x\right)^{-1}\left(t+b^{2} x\right)^{-1}$ (as a function of $\left.x\right)$. This then implies the complete monotonicity of $h^{\prime}$. Thus the function $F(x ; \mu, \nu)=e^{-h(x)}$ is also completely monotonic (see Criterion 2 in [5, p. 441]). By Bernstein's theorem [5, p. 439] we see that $F(x ; \mu, \nu)$ must be the Laplace transform of a probability distribution since $F(0 ; \mu, \nu)=1$.

Note that in the process of proving Theorem 2 we also have shown that:

COROLLARY. The function $F(x ; \mu, \nu)$, defined in (1.1), is a completely monotonic function of $x$ for $\nu \geq \mu \geq 0$ and $b>a>0$.

3. An integral representation. The purpose of this section is to establish an integral representation for the function $F$ of Theorem 2.

THEOREM 3. If $K_{\lambda}$ is the modified Bessel function of the second kind and of order $\lambda$, then

$$
\begin{aligned}
& \frac{K_{\mu}\left(b z^{1 / 2}\right) K_{\nu}\left(a z^{1 / 2}\right)}{K_{\mu}\left(a z^{1 / 2}\right) K_{\nu}\left(b z^{1 / 2}\right)}-1 \\
& \quad=\frac{1}{\pi} \int_{0}^{\infty} \frac{M(t, a, b, \mu, \nu) d t}{(z+t)\left[J_{\mu}^{2}\left(a t^{1 / 2}\right)+Y_{\mu}^{2}\left(a t^{1 / 2}\right)\right]\left[J_{\nu}^{2}\left(b t^{1 / 2}\right)+Y_{\nu}^{2}\left(b t^{1 / 2}\right)\right]}
\end{aligned}
$$


for $\mu>0, \nu>0, a>0, b>0$ where

$$
\begin{aligned}
M\left(t^{2}, a, b, \mu, \nu\right)= & {\left[J_{\mu}(a t) J_{\nu}(a t)+Y_{\mu}(a t) Y_{\nu}(a t)\right] } \\
& \times\left[J_{\nu}(b t) Y_{\mu}(b t)-J_{\mu}(b t) Y_{\nu}(b t)\right] \\
& -\left[J_{\mu}(b t) J_{\nu}(b t)+Y_{\mu}(b t) Y_{\nu}(b t)\right] \\
& \times\left[J_{\nu}(a t) Y_{\mu}(a t)-J_{\mu}(a t) Y_{\nu}(a t)\right]
\end{aligned}
$$

and $J_{\mu}, Y_{\nu}$ are the Bessel functions of the first and second kind respectively.

The proof utilizes the representation and inversion theorems for the Stieltjes transform, namely,

LEMMA (REPRESENTATION THEOREM). If

(i) $H(z)$ is analytic for $|\arg z|<\pi / \alpha$ for some $\alpha, 0<\alpha<1$, and

(ii) $H(z)=o(1)$ as $|z| \rightarrow \infty$ and $H(z)=o\left(|z|^{-1}\right)$ as $|z| \rightarrow 0$ uniformly in every sector $|\arg z|<\pi / \alpha^{\prime}$ with $\alpha^{\prime}>\alpha$, then

$$
H(z)=\frac{1}{\pi} \int_{0}^{\infty} \frac{d t}{x+t} \frac{1}{2 \pi i} \int_{C} \frac{\zeta H\left(t e^{\zeta}\right) e^{\zeta / 2}}{\varsigma^{2}+\pi^{2}} d \zeta
$$

where $C$ is a rectifiable closed curve going around $[-i \pi, i \pi]$ in the positive direction and lying in the strip $|\operatorname{Im} s|<\pi / \alpha$.

For a proof of this lemma see [6, pp. 210 and 235]. We are now in a position to prove our theorem.

Proof of Theorem 3. Denote the left-hand side of (3.1) by $H(z)$. Then $H(z)$ satisfies condition (i) of the Lemma, for some $\alpha>2 / 3$, because $K_{\lambda}(z)$ has finitely many zeros and none in the half-plane $\operatorname{Re} z \geq 0$. For $\lambda \geq 0$ we have $K_{\lambda}(z) \sim(\pi / 2 z)^{1 / 2} e^{-z}$ uniformly as $|z| \rightarrow \infty$ in the sector $|\arg z| \leq 3 / 2-\delta$ for any $\delta \in(0,3 \pi / 2)$, (see [13, p. 203]). As $|z| \rightarrow 0$ we have $K_{\lambda}(z) \sim \frac{1}{2} \Gamma(\lambda)\left(\frac{1}{2} z\right)^{-\lambda}$ for $\lambda>0$ and $K_{0}(z) \sim-\ln z$, (see [1, p. 375]). Hence $H(z)$ also satisfies condition (ii) of the Lemma.

The contour integral in (3.3) is now readily seen to be

$$
\frac{i}{2}\left[H\left(t e^{i \pi}\right)-H\left(t e^{-i \pi}\right)\right]
$$

Equation (3.1) now follows, for $z>0$, from (3.4) and

$$
K_{\nu}\left(z e^{i \pi / 2}\right)=-\frac{1}{2} i \pi e^{-i \pi \nu / 2}\left[J_{\nu}(z)-i Y_{\nu}(z)\right]
$$

and

$$
K_{\nu}\left(z e^{-i \pi / 2}\right)=\frac{1}{2} i \pi e^{i \pi \nu / 2}\left[J_{\nu}(z)+i Y_{\nu}(z)\right],
$$

(see, for example, [4, pp. 4 and 6]). Finally, (3.1) follows for $|\arg z|<\pi$ from $z>0$ by analytic continuation.

4. Discussion of conjecture. We shall show that the conjecture made in $\S 1$ is equivalent to the statement that the function

$$
\boldsymbol{\Theta}_{\nu}(x ; a, b)=\sum_{n=1}^{\infty}\left(e^{-j_{\nu, n}^{2} a x}-e^{-j_{\nu, n}^{2} b x}\right), \quad 0<a<b, \quad \nu \geq 0,
$$

is a decreasing function of $\nu$, where $j_{\nu, 1}<j_{\nu, 2}<\cdots<j_{\nu, n}<\cdots$ are the successive positive zeros of the Bessel function of the first kind and order $\nu$. 
From (1.2)

$$
\begin{aligned}
-2 x^{1 / 2} \frac{d}{d x} \ln G(x ; \mu, \nu)= & b \frac{I_{\mu+1}\left(b x^{1 / 2}\right)}{I_{\mu}\left(b x^{1 / 2}\right)}-a \frac{I_{\mu+1}\left(a x^{1 / 2}\right)}{I_{\mu}\left(a x^{1 / 2}\right)} \\
& -b \frac{I_{\nu+1}\left(b x^{1 / 2}\right)}{I_{\nu}\left(b x^{1 / 2}\right)}+a \frac{I_{\nu+1}\left(a x^{1 / 2}\right)}{I_{\nu}\left(a x^{1 / 2}\right)}
\end{aligned}
$$

where we have used $[13$, p. 79$]$

$$
I_{\lambda}^{\prime}(x)=I_{\lambda+1}(x)+\lambda I_{\lambda}(x) / x .
$$

An analog of (2.4) is the Mittag-Leffler expansion [4, p. 61]

$$
\frac{I_{\lambda+1}(z)}{I_{\lambda}(z)}=2 z \sum_{n=1}^{\infty}\left(z^{2}+j_{\lambda, n}^{2}\right)^{-1}, \quad \lambda>-1 .
$$

Combining (4.1), (4.2) and (4.3) we get

$$
\begin{aligned}
-\frac{d}{d x} G(x ; \mu, \nu)= & \sum_{n=1}^{\infty}\left[\left(x+j_{\mu, n}^{2} b^{-2}\right)^{-1}-\left(x+j_{\mu, n}^{2} a^{-2}\right)^{-1}\right] \\
& -\sum_{n=1}^{\infty}\left[\left(x+j_{\nu, n}^{2} b^{-2}\right)^{-1}-\left(x+j_{\nu, n}^{2} a^{-2}\right)^{-1}\right] \\
= & \int_{0}^{\infty} e^{-x t}\left[\Theta_{\mu}\left(t ; b^{-2}, a^{-2}\right)-\Theta_{\nu}\left(t ; b^{-2}, a^{-2}\right)\right] d t
\end{aligned}
$$

which shows that the conjecture is equivalent to the above-mentioned property of $\boldsymbol{\theta}_{\nu}(x ; a, b)$.

The sums appearing in (4.1) resemble theta functions, and, in fact, when $\nu=$ $\pm \frac{1}{2}$ the function $\theta_{\nu}$ reduces to the difference of two theta functions,

$$
\begin{aligned}
\Theta_{1 / 2}(x ; a, b) & =\frac{1}{2}\left[\vartheta_{3}(0, q)-\vartheta_{3}\left(0, q^{\prime}\right)\right], \\
\Theta_{-1 / 2}(x ; a, b) & =\frac{1}{2}\left[\vartheta_{2}(0, q)-\vartheta_{2}\left(0, q^{\prime}\right)\right],
\end{aligned}
$$

where $q=e^{-\pi^{2} a x}$ and $q^{\prime}=e^{-\pi^{2} b x}$.

\section{REFERENCES}

1. M. Abramowitz and I. A. Stegun, Handbook of mathematical functions with formulas, graphs, and mathematical tables, Appl. Math. Series No. 55, Nat. Bur. Standards, U.S. Government Printing Office, Washington, D.C., 1964.

2. R. Askey and M. E. H. Ismail, Recurrence relations, continued fractions and orthogonal polynomials (in preparation).

3. R. Askey and M. E. H. Ismail, The Rogers q-ultraspherical polynomials, Approximation Theory, edited by E. Cheney, Academic Press, New York, 1980, pp. 175-182.

4. A. Erdélyi, W. Magnus, F. Oberhettinger and F. G. Tricomi, Higher transcendental functions, vol. 2, McGraw-Hill, New York, 1953.

5. W. Feller, An introduction to probability theory and its applications, vol. II, 2nd ed., Wiley, New York, 1970.

6. I. I. Hirschman and D. V. Widder, The convolution transform, Princeton Univ. Press, Princeton, N.J., 1955.

7. M. E. H. Ismail, Bessel functions and the infinite divisibility of the Student t-distribution, Ann. Probab. 5 (1977), 582-585.

8. _ Integral representations and complete monotonicity of various quotients of Bessel functions, Canad. J. Math. 29 (1977), 1198-1207. 
9. M. E. H. Ismail and D. H. Kelker, Special functions, Stieltjes transforms and infinite divisibility, SIAM J. Math. Anal. 10 (1979), 884-901.

10. M. E. H. Ismail and C. P. May, Special functions, infinite divisibility and transcendental equations, Math. Proc. Cambridge Philos. Soc. 85 (1979), 453-464.

11. J. Kent, Some probabilistic properties of Bessel functions, Ann. Probab. 6 (1978), 760-770.

12. K. S. Miller, Hypothesis testing with complex distributions, Krieger, Huntington, N.Y., 1980.

13. G. N. Watson, $A$ treatise on the theory of Bessel functions, Cambridge Univ. Press, Cambridge, 1966.

14. J. Wendel, Hitting spheres with Brownian motion, Ann. Probab. 8 (1980), 164-169.

15. J. Wimp, Orthogonal polynomials in the tabulation of Stieltjes transforms, J. Math. Anal. Appl. (to appear).

Department of Mathematics, Arizona State University, Tempe, Arizona 85287

RIVERside Research Institute, 1701 NORTH FORT MYer DRIVE, ARLington, VIRGINIA 22209 